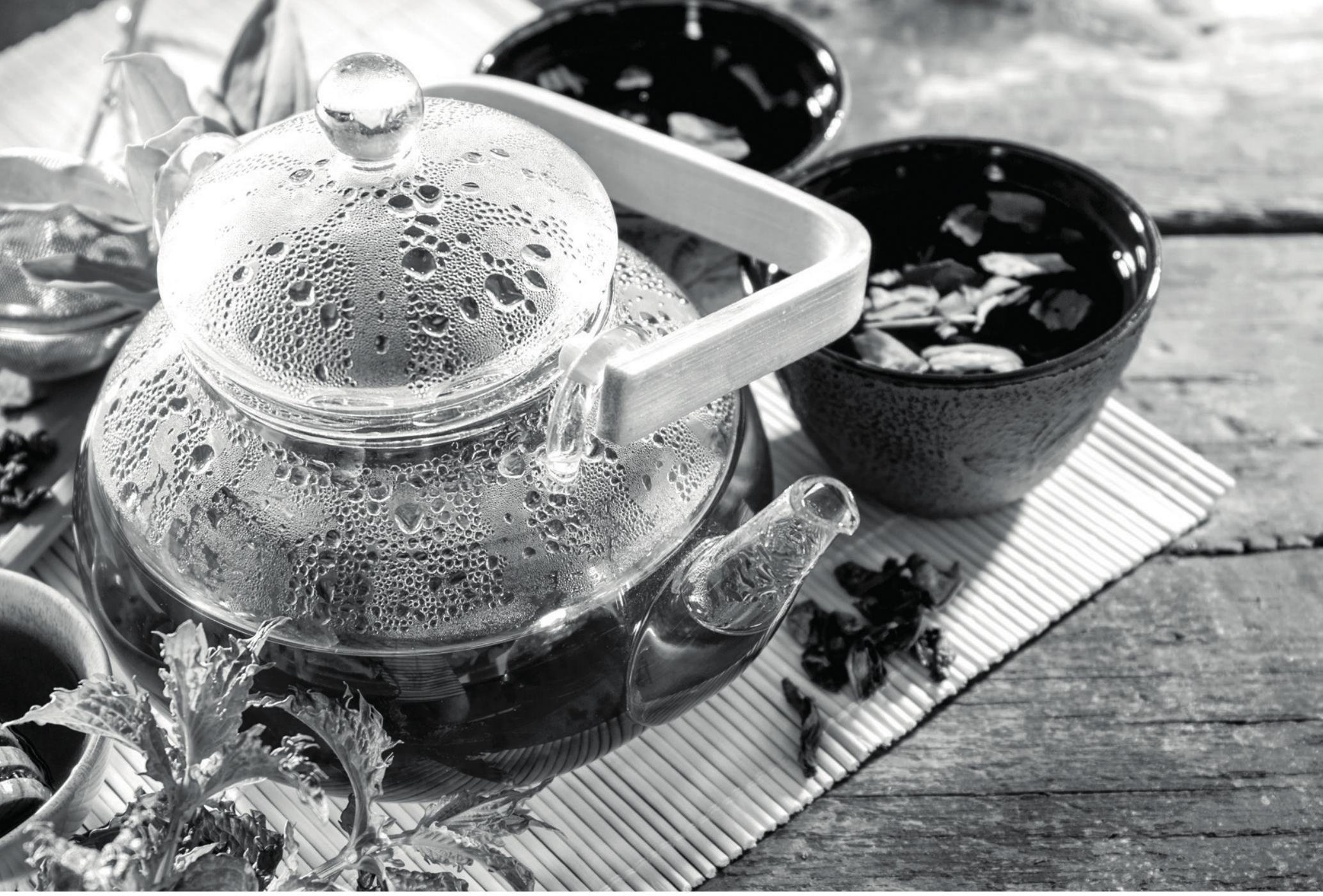

\title{
A FITOTERAPIA NO CONTEXTO DA ATENÇÃOO BÁSICA
}

Josefa Waldenora da Costa'

Karla Cristina Marques Afonso Ferreira ${ }^{2}$

Nathanny Ferreira Moutinho ${ }^{3}$

Thiago da Silva Bezerra ${ }^{4}$

Vilani Medeiros de Araújo Nunes ${ }^{5}$

\section{RESUMO}

Objetivo: Avaliar o uso de plantas medicinais e o nível de conhecimento sobre fitoterapia por gestores e gerentes das Unidades de Saúde da Família (USF), bem como dos profissionais das equipes da Estratégia de Saúde da Família e da comunidade assistida por essas equipes. Metodologia: Estudo descritivo com abordagem quantitativa realizado

em Parnamirim, RN. Foram entrevistados 596 usuários em seis USF, 08 gestores/gerentes dos serviços e 123 profissionais da saúde, entre julho e setembro de 2016. Resultados: Falta de coerência entre a espécie da planta citada pelos usuários e a doença tratada com as indicações encontradas na literatura. Entre os profissionais, 60,2\% referem pouco 
conhecimento sobre fitoterapia, 91,9\% dos gestores desconhece a Política Nacional de Plantas Medicinais e Fitoterápicos, embora $50,4 \%$ já tenham indicado tratamento à base de plantas medicinais. Conclusão: Verificouse que grande desafio na consolidação do uso de Plantas Medicinais e Fitoterápicos na qualificação dos profissionais.

Palavras-chave: Plantas Medicinais.

Medicamentos Fitoterápicos. Estratégia Saúde da Família. Atenção Básica.

\section{INTRODUÇÃo}

As plantas medicinais sempre estiveram presentes na vida da humanidade, influenciadas pela sabedoria indígena e pela medicina tradicional chinesa, que sempre as utilizou como forma preventiva e curativa de doenças. Apesar dos avanços tecnológicos e com terapias farmacológicas modernas, o uso das plantas medicinais ainda é grande, por questões de tradição, baixo custo e fácil acesso. (TITONELLI et al., 2006).

Os produtos obtidos de plantas medicinais possuem diversas definições na área farmacêutica, dependendo de sua etapa tecnológica de processamento. $\mathrm{O}$ resultado da última etapa do processamento é definido pela Agência Nacional de Vigilância Sanitária (ANVISA) como fitoterápico. Este se caracteriza como um medicamento farmacêutico obtido por processos tecnologicamente adequados, empregando exclusivamente matérias-primas vegetais, com finalidade profilática, curativa, paliativa ou para fins de diagnóstico. Além disso, tem sua eficácia e riscos de uso conhecido, assim como, reprodutibilidade e constância de sua qualidade (BRASIL, 2000).

Para Sacramento (2001), a fitoterapia sobreviveu no Brasil devido ao conhecimento enraizado na cultura e crença popular que reconheceu sua eficácia e legitimidade. Esse reconhecimento veio durante a Conferência Internacional sobre cuidados da Atenção Primária à Saúde (APS), realizada em AlmaAta (parte da antiga União das Repúblicas Socialistas Soviéticas, URSS) em 1978, onde a Organização Mundial da Saúde (OMS) declarou oficialmente legitimidade ao uso de plantas medicinais e medicamentos fitoterápicos no tratamento da saúde e recomendou a difusão dos conhecimentos necessários ao seu uso. Desde então, há uma tendência mundial de defesa, estímulo e inserção da fitoterapia nos programas APS (WHO, 2002).

No Brasil, a fitoterapia é enquadrada pelo Sistema Único de Saúde (SUS), como prática integrativa e complementar à saúde (BRASIL, 2006). Tais práticas se inserem no que a OMS denomina de medicina tradicional (MT) e medicina complementar e alternativa (MCA) e, sobre esse tema, a OMS recomenda aos seus Estados membros a elaboração de políticas nacionais voltadas à integração ou à inserção da MT/MCA aos sistemas oficiais de saúde, com foco na Atenção Primária à Saúde.

Atualmente, o uso da fitoterapia como opção terapêutica tem respondido bem ao tratamento de muitas patologias comuns na Atenção Primária à Saúde (BERTOLDI et al., 2004). Fontenele et al. (2013) relatam que a fitoterapia se tornou mais consistente na Atenção Básica a partir da constatação de que a população atendida pelas USF faz uso de plantas medicinais com fins terapêuticos, simultaneamente, ao uso de medicamentos industrializados. Em contrapartida o uso concomitante destes medicamentos ocorre, na maioria das vezes, sem o conhecimento pleno sobre a toxicidade e ação terapêutica das plantas medicinais por parte dos usuários.

Pesquisas apontam que muitos profissionais de saúde desconhecem sobre as terapias 
complementares, apesar de se interessarem pelo tema e de aprovarem sua inclusão nos serviços públicos de saúde (SACRAMENTO, 2001). E os profissionais de saúde no contexto da USF são importantes agentes, com grande responsabilidade nas ações diagnósticas e de orientação preventiva e terapêutica no cuidado da saúde da população (THIAGO; TESSER, 2010).

A importância da realização de estudos sobre o cultivo, uso das plantas medicinais, indicação, posologia e modo de preparo é de grande relevância, considerando a segurança na sua utilização. A partir de informações obtidas nas pesquisas, pode-se identificar quais são as principais plantas utilizadas, indicações quanto ao uso correto baseado em estudos científicos e como se dá a relação do profissional com a indicação e o uso desse tipo de terapia complementar pela população.

Dessa forma, o presente estudo teve como objetivo avaliar o perfil de utilização de plantas medicinais e as patologias tratadas pelas equipes da Estratégia de Saúde da Família (ESF) nas Unidades Básicas de Saúde (UBS), bem como o conhecimento dos profissionais, gestores e gerentes do município de Parnamirim, $\mathrm{RN}$, abrangendo todos os principais aspectos voltados ao tema.

\section{METODOLOGIA}

Trata-se de um estudo descritivo com abordagem quantitativa realizado no município de Parnamirim/RN com os usuários, profissionais, gestores e gerentes das Unidades Básicas de Saúde acerca do conhecimento das práticas integrativas e complementares em saúde, em relação ao uso de plantas medicinais e fitoterápicos. $\mathrm{O}$ estudo foi realizado em três etapas, considerando o levantamento bibliográfico, realização de entrevistas, interpretação e análises dos dados.

A revisão da literatura sobre o tema do estudo foi realizada a partir da busca em artigos, livros, revistas e sites especializados na perspectiva de melhor conhecimento sobre o tema e, por conseguinte utilizá-los como base na elaboração da análise, caracterizando-se como o primeiro momento do estudo.

$\mathrm{Na}$ segunda etapa, ocorreu a coleta de dados, realizada entre os meses de julho a setembro de 2014. Nesse período, foram feitas entrevistas individuais seguindo um formulário previamente elaborado e especificado para as diferentes categorias de participantes: usuários, profissionais, gestores e gerentes de serviços, contendo questões fechadas com a finalidade de caracterizar o perfil destes em relação ao grau de conhecimento sobre plantas medicinais e fitoterápicos, formas de utilização e cultivo, partes das plantas que são usadas, conhecimento sobre a Política Nacional de Plantas Medicinais e fitoterápicos e implantação no município das Práticas Integrativas e Complementares em Saúde (PICS) na Atenção Básica.

O município de Parnamirim/RN possui uma cobertura de 100\% das UBS inseridas na ESF e de acordo com dados da Secretaria Municipal de Saúde (SMS), no ano do estudo, contava-se com 25 UBS divididas em seis polos. Para realização do presente estudo foram selecionadas seis UBS, correspondendo à representação de cada polo de saúde no município, a partir da realização de um sorteio aleatório por cada polo. Excepcionalmente, no polo VI, não foi selecionada nenhuma unidade porque este congrega os Centros de Apoio Psicossocial (CAPS), que não se enquadram no perfil desta pesquisa.

Uma outra exceção foi o polo $\mathrm{V}$, no qual foram selecionadas duas UBS, Liberdade e Pium, essa última por estar inserida em uma área litorânea e rural, sendo a única área do município com estas características. Dessa forma, as unidades selecionadas por polo foram: 
Polo I: UBS Parque Industrial; Polo II: UBS Centro; Polo III: UBS Passagem de Areia; Polo IV: UBS Santa Tereza; Polo V: UBS Liberdade e UBS Pium.

Participaram do estudo 595 usuários, 123 profissionais da ESF, entre eles, médicos, dentistas, enfermeiros, técnicos de enfermagem, técnicos em saúde bucal (TSB), agentes comunitários em saúde (ACS), além de cinco diretores das unidades de saúde, a coordenadora geral da Atenção Primária de Saúde, a coordenadora da ESF e o Secretário Municipal de Saúde de Parnamirim, os quais aceitaram participar de forma voluntária, assinando o Termo de Consentimento Livre e Esclarecido (TCLE).

A coleta de dados realizada nas UBS foi agendada em consonância com a disponibilidade da gestão da SMS/Parnamirim, dos gerentes dos serviços e de acordo com os dias em que a unidade apresentasse o maior fluxo de atendimento aos usuários para que o maior número de pessoas da comunidade pudesse participar do estudo.

Os critérios utilizados para seleção dos participantes da comunidade foram: ser usuário do serviço; ter idade igual ou superior a 18 anos e, não ser portador de doença psicocognitiva que impedisse o entendimento do estudo. Os questionários foram aplicados individualmente para cada usuário que se encontrava na UBS, na sala de espera enquanto aguardava o atendimento. Entre as variáveis de estudo respondidas estão: sexo, nível de escolaridade, se fazem uso de plantas medicinais, quais plantas são cultivadas (para os que cultivam), a forma de utilização dessas plantas e Política Nacional de Práticas Integrativas e Complementares.

Para a realização dos testes de associação do qui-quadrado $\left(\chi^{2}\right)$ utilizou-se o programa SPSS - Statistical Package for the Social Sciences versão 22.0. O teste do qui-quadrado é utilizado para saber o nível de significância, se for menor que 0,05, o teste é significativo e existe associação entre as variáveis.

A terceira etapa consistiu na interpretação e análises dos dados. Para tabular e apresentar os dados dos formulários, foi empregado o programa Microsoft Excel®2010 que serviu como auxílio para fazer estatística simples, permitindo a apresentação dos dados em gráficos e tabelas. As análises estatísticas foram processadas pelo software Statistical Package for Social Sciences (SPSS), versão 22.

A presente pesquisa se deu a partir de um projeto de extensão intitulado Quintais Medicinais: diagnóstico sobre o cultivo e consumo de plantas medicinais por famílias atendidas pela Estratégia Saúde da Família. Inicialmente, o projeto foi apresentado à Secretaria Municipal de Saúde de Parnamirim para obtenção da autorização, por meio da carta anuência, para a realização da pesquisa nas Unidades Básicas de Saúde (UBS) do município. O estudo foi aprovado pelo Comitê de Ética em Pesquisa da Universidade Federal do Rio Grande do Norte (CEP/UFRN), sob registro do CONEP/CNS n ${ }^{\circ}$ 003/2011, CAAE n 31746114.9.0000.5292 e Parecer de Aprovação sob n 732.426. 


\section{RESULTADOS}

CARACTERIZAÇÃO DOS USUÁRIOS DAS UBS

DA ESTRATÉGIA SAÚDE DA FAMÍLIA

De acordo com a Tabela 1, foi observado que as plantas medicinais são utilizadas por boa parte da população estudada para o tratamento de diversas doenças e agravos à saúde. A fitoterapia foi mais citada com recurso terapêutico para o tratamento de má digestão $(28,7 \%)$, gripe $(16,8 \%)$ e estresse $(15,9 \%)$.
UTILIZAÇÃO DA FITOTERAPIA PELOS USUÁRIOS DA UBS

De acordo com a Tabela 1, foi observado que as plantas medicinais são bastante utilizadas pela comunidade em estudo no combate a doenças como má digestão $(28,7 \%)$, gripe $(16,8 \%)$ e estresse $(15,9 \%)$.

Tabela 1 - Principais sintomas/doenças listados pela população que fazem 0 tratamento com plantas medicinais.

\begin{tabular}{|ccc|}
\hline Sintoma/Doença & No Observações & Percentual \\
\hline Má digestão* $^{*}$ & 347 & $28,7 \%$ \\
\hline Gripe* $^{*}$ Estresse/Calmante* $^{*}$ & 203 & $16,8 \%$ \\
\hline Outras & 192 & $15,9 \%$ \\
\hline Tosse & 87 & $7,2 \%$ \\
\hline Inflamação & 57 & $4,7 \%$ \\
\hline Dor (geral) & 39 & $3,2 \%$ \\
\hline Dor de garganta & 31 & $2,6 \%$ \\
\hline Mal-estar & 27 & $2,2 \%$ \\
\hline Doenças de pele & 26 & $2,2 \%$ \\
\hline Hipertensão & 21 & $1,7 \%$ \\
\hline Obesidade & 21 & $1,7 \%$ \\
\hline Colesterol & 19 & $1,6 \%$ \\
\hline Dor de cabeça & 19 & $1,6 \%$ \\
\hline Dor na coluna & 18 & $1,5 \%$ \\
\hline Diarreia & 16 & $1,3 \%$ \\
\hline Insônia & 14 & $1,2 \%$ \\
\hline Gastrite & 14 & $1,2 \%$ \\
\hline Dor no ouvido & 13 & $1,1 \%$ \\
\hline Febre & 12 & $1,0 \%$ \\
\hline Vermes & 11 & $0,9 \%$ \\
\hline Diabetes & 10 & $0,8 \%$ \\
\hline Total & 10 & $0,8 \%$ \\
\hline & 1207 & \\
\hline & & $100 \%$ \\
\hline
\end{tabular}

Fonte: Elaborado pelo próprio autor (2014). 
As plantas mais citadas foram: hortelã $(14,2 \%)$, capim santo $(8,2 \%)$, erva-doce $(5,0 \%)$, babosa $(3,3 \%)$, camomila $(6,6 \%)$, boldo $(14,2 \%)$, erva-cidreira $(8 \%)$, mastruz $(7,2 \%)$, romã $(3,5 \%)$ e louro $(2,4 \%)$ - Tabela 2.

A forma de utilização dessas plantas é importante não somente para a garantia de presença do princípio ativo, mas também, para a certificação de baixa toxicidade. (AOYAMA; INDRIUNAS; FURLAN, 2011). Verificou-se que, a principal forma de preparo se dá por meio de remédios caseiros populares como chás (74\%), seguido de xarope $(32,2 \%)$ e lambedor $(22 \%)$. Com relação às partes mais utilizadas das plantas, foram encontradas que as mais utilizadas são as folhas (81\%), seguido das cascas $(6,2 \%)$ e raízes $(3,1 \%)$.

\section{Tabela 2 - Plantas medicinais utilizadas pelos entrevistados da UBS do município de Parnamirim/RN.}

\section{Plantas}

\begin{tabular}{ccc|}
\hline Outras & 237 & $18,7 \%$ \\
\hline Hortelã (Menthapiperita L.) & 180 & $14,2 \%$ \\
\hline Boldo (Peumus boldus Molina L.) & 179 & $14,1 \%$ \\
\hline Capim-santo (Cymbopogon citratus L.) & 103 & $8,1 \%$ \\
\hline Erva-cidreira (Melissa officinalis L.) & 101 & $8,0 \%$ \\
\hline Mastruz (Chenopodium & & \\
ambrosioides L.) & 91 & $7,2 \%$ \\
\hline Camomila (Chamomilla recutita L.) & 84 & $6,6 \%$ \\
\hline Erva-doce (Pimpinella anisum L.) & 63 & $5,0 \%$ \\
\hline Romã (Punica granatum L.) & 44 & $3,5 \%$ \\
\hline Babosa (Aloe vera L.) & 42 & $3,3 \%$ \\
\hline Louro (Laurus nobilis L.) & 30 & $2,4 \%$ \\
\hline Chá-verde (Cammelia sinensis L.) & 21 & $1,7 \%$ \\
\hline Arruda (Ruta graveolens L.) & 20 & $1,6 \%$ \\
\hline Alecrim (Rosmarinus officinalis L.) & 17 & $1,3 \%$ \\
\hline Limão (Citrus Limonium L.) & 16 & $1,3 \%$ \\
\hline Noni (Morinda Citrifolia L.) & 14 & $1,1 \%$ \\
\hline Anador (Justicia pectoralis L.) & 14 & $1,1 \%$ \\
\hline Chá-preto (Camellia sinensis L.) & 14 & $1,1 \%$ \\
Total & 1270 & $100 \%$ \\
\hline
\end{tabular}

Fonte: Elaborado pelo próprio autor (2014).

Frequência Absoluta Frequência Relativa 


\section{PLANTAS CULTIVADAS PELOS USUÁRIOS DAS UBS}

No presente estudo foram descritos o cultivo de 73 plantas, sendo 21 destas as mais citadas pelos participantes; o cultivo dessas plantas vem de indicação dos familiares $(86,7 \%)$, corroborando com a literatura de que esses hábitos são passados ao longo das gerações.

Outro dado observado é que, quanto maior a idade, maiores as chances dessas pessoas cultivarem uma planta medicinal em casa, já que o nível de significância é de $\mathrm{x}^{2}=0,011(<0,05$ o teste é significativo e existe associação entre as variáveis), comparado as idades entre 18 aos 38 anos versus maiores que 38 anos. Dessa forma, maiores de 38 anos fazem o maior cultivo das plantas medicinais com uma frequência de $55,3 \%$ do total da população entrevistada em relação à faixa etária entre 18 e 38 anos com a frequência de $44,7 \%$.

\section{CARACTERIZAÇÃO DOS PROFISSIONAIS DAS UBS DA ESTRATÉGIA SAÚDE DA FAMÍLIA E ANÁLISE DO NIVEL DE CONHECIMENTO SOBRE $O$ ASSUNTO}

A população desse estudo constituiu-se por 123 profissionais, sendo a maioria do sexo feminino $(77,2 \%)$, nível médio $(72,4 \%)$, numa faixa de idade que variou dos 21 aos 67 anos de idade, tendo o maior percentual de profissionais entre 31 a 40 anos de idade $(49,6 \%)$. Estes estavam distribuídos em seis Unidades Básicas do município, concentrando-se um maior número de profissionais participantes na Unidade de Passagem de Areia (30,1\%); assim como a amostra constituiu-se, em sua maioria, de Agentes Comunitários de Saúde - ACS $(65,9 \%)$.

Quando analisados os dados acerca do questionamento sobre o nível de conhecimento da fitoterapia de acordo com cada distrito e com a proporcionalidade da quantidade de profissionais que atuam em cada UBS, apenas nas unidades de saúde dos bairros de Santa Tereza,
Gentro e Parque Industrial apresentaram profissionais que afirmaram ter conhecimento "ótimo" sobre a prática, sendo 1 profissional em cada UBS, totalizando $2,4 \%$ da amostra. Dos demais profissionais participantes do estudo, observou-se que $32(26,0 \%)$ classificaram o seu nível de conhecimento como "bom", $14(11,4 \%)$ afirmaram que desconheciam esta prática complementar e $74(60,2 \%)$ disseram ter pouco conhecimento sobre o assunto.

Apesar de 78,9\% dos profissionais considerarem o uso de plantas medicinais e fitoterapia como "muito importante", apenas 50,4\% revelou indicar algum tratamento à base de plantas medicinais. Diante desta concepção predominante de que tal tratamento é "muito importante", poderia se esperar um número maior de profissionais que indicassem o referido tipo de terapia em saúde.

Dentre os profissionais participantes da pesquisa, $84,6 \%$ alegaram saber o significado de fitoterapia, sendo $60,2 \%$ com pouco conhecimento e $26,0 \%$ com bom conhecimento na área; 72,4\% relataram já ter feito uso de plantas medicinais como terapia alternativa de saúde, bem como $50,4 \%$, já indicou tratamentos à base de plantas medicinais, muito embora 89,4\% relataram nunca terem feito cursos na área de PICS. Partindo do exposto, vê-se a necessidade de capacitação das equipes do ESF para o uso e manejo das plantas medicinais, tendo os gestores e órgãos formadores de profissionais fundamental importância na capacitação destes.

\section{CARACTERIZAÇÃO DOS GESTORES \\ MUNICIPAIS E GERENTES DAS UBS}

Dos gestores entrevistados, $62,5 \%$ são do sexo feminino, como também, 62,5\% possuem acima de 40 anos. Com relação à escolaridade, $75 \%$ dos pesquisados possuem grau superior completo. 
CONHECIMENTO DOS GESTORES E GERENTES

DOS POLOS DA ESF SOBRE A UTILIZAÇÃO DE

PLANTAS MEDICINAIS COMO FORMA

\section{DE MEDICINA ALTERNATIVA}

A maioria dos entrevistados, ou seja, 05 dos 08 utilizam as plantas medicinais como forma de Medicina Alternativa, reconhece os benefícios da terapêutica à qualidade de vida das pessoas embasadas em crenças populares.

\section{CONHECIMENTO DOS GESTORES E GERENTES DOS} POLOS DA ESF SOBRE A POLÍTICA NACIONAL DE PRÁTICAS INTEGRATIVAS E COMPLEMENTARES

Dos 08 participantes da pesquisa, 04 têm "pouco conhecimento", 03 têm um "médio conhecimento" e 01 "não conhece". Apesar dos dados coletados apresentarem 03 dos participantes com um "médio conhecimento" da Política Nacional de Práticas Integrativas e Complementares, percebe-se que nenhum embasou em suas palavras participações em capacitações sobre práticas integrativas e complementares, além de não conhecerem as normatizações/portarias, apenas terem ouvido falar. Dessa forma, a falta de conhecimento técnico dificulta a propagação do conhecimento das políticas e portarias.

De acordo com os oito entrevistados, o município de Parnamirim/RN não implantou as PICS na ESF. Estes acreditam que para Implantação das PICS no município é necessária uma série de requisitos como: capacitação de profissionais, comprometimento político e utilização de indicadores.

\section{DISCUSSÕES}

É importante enfatizar que a realidade de grande parte da população brasileira, ainda hoje, é marcada pela precariedade e desigualdade no que diz respeito ao acesso aos medicamentos e tratamentos médicos necessários, característica predominante nos usuários da Atenção Básica. Por esse motivo a comunidade tem buscado terapias alternativas, entre as quais se destacam o uso de plantas medicinais e fitoterápicos, afim de alcançar melhor qualidade de saúde (SAMPAIO et al., 2013).

De maneira geral, observa-se a falta de prescrição por parte dos profissionais das ESF e de orientação quanto ao uso correto, provavelmente devido ao pouco conhecimento dos profissionais da área de saúde. Isso traz prejuízos à população, uma vez que poderiam utilizar recursos eficazes e acessíveis com maior frequência e com maior segurança (SILVA et al.,2012).

De acordo com os resultados do estudo, foi observado que o uso de algumas plantas medicinais não tinha a mesma recomendação que a indicada pela literatura. O capim-santo destacou-se, pois a maioria dos entrevistados citou que servia para dor na coluna e febre, no entanto, seu uso é apropriado para cólicas intestinais e uterinas e quadros leves de ansiedade e insônia e como calmante suave (ELDIN ; DUNFORD, 2001). Por ser a terceira espécie mais citada pelos entrevistados se deve ter o cuidado ao fazer uso dela, pois se utilizada junto com medicamentos sedativos potencializa ainda mais o efeito (BRASIL, 2011).

O Chenopodium ambrosioides L. (mastruz) apesar de ser amplamente usado na medicina popular para o tratamento de pneumonia, poderá trazer riscos às crianças, pois o óleo essencial em altas doses pode provocar náuseas, vômitos, depressão respiratória, lesões hepáticas e renais, transtornos visuais, convulsões, coma e insuficiência cardiorrespiratória, sendo ainda contraindicado em crianças abaixo dos três anos (ALONSO, 1998). Hortelã (Menthapiperita L.) é a planta mais cultivada pela população, em que $151(25,33 \%)$ entrevistados afirmaram possuir em suas casas. Essa erva é utilizada na forma de infusão e também os óleos essenciais que podem ser extraídos da planta. Popularmente é indicada como estimulante gástrico nas atonias digestivas, flatulências, vômitos, vermífugo, cólicas uterinas, 
expectorante, além de outras indicações. Essa amplitude de indicações informais passadas de gerações em gerações por meio dos séculos é mantida atualmente (JÚNIOR; LEMOS, 2012).

A segunda mais cultivada, capim-santo (Cymbopogan citratus L.), foi citada por 111 $(18,62 \%)$ entrevistados, tendo como indicações para cefaleia de origem tensional, ansiedade, nervosismo, insônia, flatulência (gases intestinais), e como relaxante muscular - dores e tensões musculares de etiologia diversa, hipertensão arterial (RIBEIRO; DINIZ, 2008).

A melissa (Melissa officinalis L.), cultivada por $16,9 \%$ dos entrevistados, é conhecida como erva-cidreira verdadeira e também possui outros nomes populares como chá de frança, cidrilha, chá de tabuleiro, citronela, citronela-menor, erva-luísa, limonete, meliteia, salva-do-brasil e melissa romana (MEIRA; SOUZA; MARTINS, 2014). Seus principais constituintes de interesse medicinal e condimentar, como citral, citronelal e geraniol, entre outros, encontram-se em seu óleo essencial obtido principalmente das folhas, as quais proporcionam rendimentos de 0,02 a $0,37 \%$. Outros constituintes na planta são os ácidos hidroxicinâmicos, como o ácido rosmarínico, e os flavonoides e os taninos (AOYAMA ; INDRIUNAS ; FURLAN, 2011).

Chenopodium ambrosioides L., cultivada por 10,4\% dos entrevistados, é conhecida na medicina folclórica como erva-de-santa-maria e mastruço, é indicada como anti-inflamatória, peitoral, estomáquica, antituberculosa, béquica e vulnerária. No meio rural, a erva-de-santa-maria é empregada como repelente de ectoparasitos como piolhos, pulgas e carrapatos. Suas propriedades anti-helmínticas são apregoadas na tradição oral com referência ao combate de vermes intestinais, tais como ascarídeos, ancilostomídeos e oxiurídeos (BORBA; AMORIM, 2004).

A espécie Peumus boldus L., cultivada por 8,4\% dos usuários das UBS de Parnamirim, é bastante difundida na cultura medicinal brasileira e, frequentemente, encontrada na composição de fitoterápicos industrializados, além de ser comercializada em feiras livres em todo território nacional (MELO et al., 2004). As árvores de P. boldos plantadas no Brasil não produzem princípios ativos (boldina) suficientes para justificar seu emprego. É importante observar que os estudos toxicológicos sugerem que o consumo de chá de boldo deva ser feito com moderação e cuidado, principalmente no primeiro trimestre da gravidez e no uso por tempo prolongado uma vez que há grandes indícios de teratogenia e hepatotoxidade. $\mathrm{Na}$ realidade, o chá dessa espécie deve ser proibido para gestante (RUIZ et al., 2011).

A aloe vera L., cultivada por 7,8\% dos pesquisados, é originária da região Mediterrânea e popularmente conhecida como babosa-verdadeira, aloe-de-bardados e aloe-de-curaçau, é conhecida popularmente no Brasil como babosa e tem sido afirmado possuir várias propriedades terapêuticas importantes incluindo a aceleração da cicatrização de feridas (RAMOS; PIMENTEL, 2011). O emprego de plantas medicinais com finalidade terapêutica vem de muitos anos, está ligada a tradições e costumes da própria população que vai sendo repassada de geração em geração (TEIXEIRA et al., 2014).

A correlação da maior proporção do cultivo nas pessoas que apresentam maior idade evidencia a crença nas terapias ditas "naturais" e no conhecimento adquirido ao longo dos anos, que é passado de geração a geração (MOTOMIYA et al., 2004).

As plantas mais cultivadas pelos entrevistados que frequentam as UBS de Parnamirim-RN são: hortelã, capim-santo, erva-cidreira, mastruz, boldo e babosa. Estas são largamente utilizadas por serem de conhecimento popular e de cultivo simples, sendo elas característica de uso na região Nordeste, confirmada pelo trabalho de Mosca \& Loiola (RIBEIRO; DINIZ, 2008).

Este resultado pode ser explicado pela falta de conhecimento dos profissionais sobre o assunto, indicando que, provavelmente, estes não 
tenham recebido a devida abordagem durante a formação acadêmica ou que não realizaram algum curso específico na área (BORBA; AMORIM, 2004).

Thiago e Tesser (2010) também afirmaram em sua pesquisa que para a maioria dos profissionais do presente estudo ocorrido em Florianópolis, as PICS estão baseadas no entendimento mais amplo do processo saúde-doença, o que pode estar relacionado ao seu interesse por essas práticas. Os resultados desse estudo também indicaram baixo conhecimento dos profissionais sobre o assunto, o que sugere a necessidade de capacitações e de divulgação desses temas para implantação da Política Nacional de Práticas Integrativas e Complementares (PNPIC).

Em contrapartida, apenas 14 profissionais que afirmaram "desconhecer" a fitoterapia, porém fazem uso de plantas medicinais. Assim como, dos 74 profissionais que asseguraram "conhecer pouco" sobre fitoterapia, apenas 48 alegaram utilizar plantas medicinais. Uma das hipóteses para esse resultado é a comercialização de plantas medicinais em farmácias e lojas de produtos naturais e, dessa forma, a população em geral tem livre acesso a esses produtos, sem ter conhecimento sobre a comprovação de suas propriedades farmacológicas, que são propagadas por usuários ou comerciantes (VEIGA et al., 2005). Por isso, a importância da capacitação nos serviços de saúde sobre esse tema (BRASIL, 2012). Em vista disso, a formação na área das Práticas Complementares pode colaborar para que os profissionais se sintam seguros, e acreditem na possibilidade de inserção das PICS no SUS.

De acordo com Furnham e Bragrath (1993), o uso de plantas com fins medicinais vem de uma população insatisfeita, muitas vezes devido a reações adversas causadas pelos medicamentos; ou ainda da busca por soluções alternativas para as doenças. Segundo relato dos entrevistados, esse uso teve como fonte de informações o contato com grupos culturais compreendendo parentes, amigos, vizinhos e os próprios usuários do SUS.

Segundo Rosa et al. (2011), um estudo semelhante realizado com médicos no estado do Rio Grande do Sul traz dados que corroboram com esta realidade. Paralelamente ao grande número de profissionais de saúde que faz uso próprio de plantas medicinais e/ou fitoterápicos, encontrou-se que a maioria utiliza, porém, não informa ao usuário a importância que é a utilização da fitoterapia.

As PICS objetivam estimular a implantação de novos programas no SUS, com melhoria do acesso da população a produtos e serviços seguros e de qualidade; sensibilizar e orientar gestores e profissionais de saúde na formulação e implantação de políticas, programas e projetos; e estruturar e fortalecer a Atenção Básica em fitoterapia, com ênfase na Atenção Básica/ Saúde da Família (BRASIL, 2012).

É de grande importância que toda a equipe tenha conhecimento sobre a fitoterapia, para que possa orientar de uma forma clara os benefícios e os malefícios da utilização do medicamento, além de informar aos usuários sobre o uso correto.

O estudo apresentou algumas limitações, tendo em vista as dificuldades na realização das entrevistas com os participantes nas três dimensões (usuários, profissionais de saúde e gestores), considerando as diferentes inserções em que se encontravam durante o desenvolvimento da pesquisa. No entanto, foram minimizadas a partir das adequações as distintas realidades. O acesso aos diferentes locais também ocasionou um tempo de espera maior para que todos pudessem participar do estudo, considerando a dificuldade de meios de transportes para se chegar aos serviços. É notória a importância da iniciativa e da mobilização de gestores/gerentes, profissionais e usuários como fatores fundamentais na concretização das ações, na superação dos desafios para a implantação das PICS nos cinco polos da ESF no município de Parnamirim/RN. 


\section{CONCLUSÃO}

A partir das informações obtidas pelos entrevistados, percebe-se que o grande desafio para a consolidação do uso de plantas medicinais e fitoterápicos no município de Parnamirim/RN, além da qualificação dos profissionais, é a necessidade de uma estrutura física adequada, recursos financeiros para implantação e manutenção de uma política de valorização destas práticas alternativas e complementares em saúde e criação e divulgação de programas e campanhas. Dessa forma, o estudo verificou que os profissionais entrevistados se mostraram interessados em buscar maiores conhecimentos sobre fitoterapia, confirmando alta aceitabilidade à utilização deste tipo de terapêutica. Verificou-se assim que a população do estudo acredita na sua eficácia, conceito esse que abrange outros aspectos como baixo custo e fácil acesso.

\section{PHYTOTHERAPY IN PRIMARY HEALTH CARE}

\section{ABSTRACT}

Objective: To evaluate the use of medicinal plants and the level of knowledge about phytotherapy by managers and managers of the Family Health Units (FHU), as well as the staff of the Family Health Strategy teams and the community assisted by these teams. Methodology: A descriptive study with a quantitative approach carried out in Parnamirim, RN. 596 users were interviewed in six FHUs, 08 managers / managers of the services and 123 health professionals, between July and September 2016. Results: Lack of coherence between the species of the plant mentioned by the users and the disease treated with the indications found in the literature. Among the professionals, 60.2\% reported little knowledge about phytotherapy, $91.9 \%$ of the managers are unaware of the National Policy of Medicinal Plants and Phytotherapics, although 50.4\% have already indicated treatment based on medicinal plants. Conclusion: It was verified that great challenge in the consolidation of the use of Medicinal Plants and Phytotherapy in the qualification of the professionals.

Keywords: Medicinal Plants. Herbal medicines. Family health strategy. Primary Care. 


\section{FITOTERAPIA EN EL CONTEXTO DE LA ATENCIÓN BÁSICA RESUMEN}

Objetivo: Evaluar el uso de plantas medicinales y el nivel de conocimiento sobre fitoterapiaporgestoresygerentesdelas Unidades de Salud de la Familia (USF), así como de los profesionales de los equipos de la Estrategia de Salud de la Familia y de la comunidad asistida por esos equipos. Metodología: Estudio descriptivo con abordaje cuantitativo realizado en Parnamirim, RN. Se entrevistó a 596 usuarios en seis USF, 08 gestores / gerentes de servicios y 123 profesionales de la salud, entre julio y septiembre de 2016. Resultados: Falta de coherencia entre la especie de la planta citada por los usuarios y la enfermedad tratada con las indicaciones encontradas en la literatura. Entre los profesionales, el 60,2\% refiere poco conocimiento sobre fitoterapia, el 91,9\% de los gestores desconoce la Política Nacional de Plantas Medicinales y Fitoterápicos, aunque el $50,4 \%$ ya ha indicado tratamiento a base de plantas medicinales. Conclusión: Se verificó que gran desafio en la consolidación del uso de Plantas Medicinales y Fitoterápicos en la calificación de los profesionales.

Palabras clave: Plantas medicinales. Las hierbas medicinales. Estrategia de Salud. Atención Primaria.

\section{REFERÊNCIAS}

ALONSO, J.R. Tratado de fitomedicina: bases clínicas Y farmacológicas. Buenos Aires: ed. Isis, $1998.1039 \mathrm{p}$.

AOYAMA, E. M; INDRIUNAS, A.; FURLAN, M. R. Produção de folhas em Melissa officinalis L. (Lamiaceae) em Taubaté, São Paulo. Revista biociências, UNITAU. v. 17, n. 1, p. 57-65, 2011.

BERTOLDI, A. D. et al. Utilização de medicamentos em adultos: prevalência e determinantes individuais. Rev Saúde Pública. n. 38, p. 228-238, 2004.

BORBA, R.H, AMORIM, A. Ação anti-helmíntica de plantas xiv. avaliação da atividade de extratos aquosos de chenopodium ambrosioides l. (erva-de-santa-maria) em camundongos naturalmente infectados com syphacia obvelata e aspiculuris tetraptera. Rev. Bras. Parasitol. Vet., v. 13, n. 4, p. 133-136, 2004.

BRASIL, Ministério da Saúde. Relatório do $1^{\circ}$ Seminário Internacional sobre Práticas Integrativas e Complementares. Brasília, 2008b. Disponível em: <http://dtr2004.saude. gov.br/dab/semi_praticas_integrativas. php>. Acesso em: 16 abr. 2015. 
BRASIL. Agência Nacional de Vigilância Sanitária. Formulário de Fitoterápicos da farmacopeia Brasileira/Agência Nacional de Vigilância Sanitária Brasileira. ANVISA, 2011.

BRASIL. ANVISA. Resolução RDG nº 17, de 24 de fevereiro de 2000. Dispõe sobre o registro de medicamentos fitoterápicos. Diário Oficial da República Federativa do Brasil, Brasília, DF: 2000.

BRASIL. Ministério da Saúde. Portaria n. 971, de 3 de maio de 2006. Aprova a Política Nacional de Práticas Integrativas e Complementares (PNPIC) no Sistema Único de Saúde. Diário Oficial [da] República Federativa do Brasil, Brasília, DF, 04 maio 2006.

BRASIL. Ministério da Saúde. Secretaria de Atenção à Saúde. Departamento de Atenção Básica. Práticas integrativas e complementares: plantas medicinais e fitoterapia na Atenção Básica/Ministério da Saúde. Secretaria de Atenção à Saúde. Departamento de Atenção Básica. - Brasília: Ministério da Saúde, 2012.

ELDIN S.; DUNFORD, A. Fitoterapia na atenção primária à saúde. São Paulo: Manole, 2001.

FONTENELE, R. P. et al. Fitoterapia na atenção básica: olhares dos gestores e profissionais da Estratégia em Saúde da Família de Terezina (PI), Brasil. Revista de Ciência Saúde Coletiva. v.18, n. 8, p. 2385-2394, 2013.

FURNHAM, A; BHAGRATH, R. A comparison of health beliefs and behaviours of clients of orthodox and complementary medicine. Br J Clin Psychol, 1993.

LEMOS JÚNIOR, L. P. H.; LEMOS, A. L. Hortelã. Universidade Federal de São Paulo Escola Paulista de Medicina (UNIFESP-EPM). Diagnóstico Tratamento, v. 17, n. 3, p. 115-117, 2012.

MEIRA, M. R.; SOUZA, S. A. M; MARTINS, E. R. Plantas medicinais: produção e cultivo da Melissa officinalis no Brasil. 2010. Disponível em: <http://www.conhecer.org.br/enciclop/2010b/plantas>. Acesso em: 10 nov. 2014.

MELO, J. G et al. Avaliação da qualidade de amostras comerciais de boldo (Peumus boldus Molina), pata-de-vaca (Bauhinia spp.) e gingo (Ginkgo biloba L.). Rev Bras Farmacogn. v. 14, n. 2, p. $111-120,2004$.

MOSCA, V.P.; LOIOLA, M.I.B. Uso popular de plantas. Rev. Bras. P1. Med., Campinas, v. 15, n. 3, p. 442-458, 2013. 
MOTOMIYA, A.V.A et al. Levantamento e Cultivo das Espécies de Plantas Medicinais Utilizadas em Cassilândia, MS. In: CONGRESSO BRASILEIRO DE EXTENSÃO UNIVERSITÁRIA BELO HORIZONTE, 2., 2004, Belo Horizonte. Anais... Belo Horizonte, 2004.

RAMOS, A. P.; PIMENTEL, L.C. Ação da babosa no reparo tecidual e cicatrização. BrazJ of Healt, v. 2, n. 1, p. 40-48, 2011.

RIBEIRO, P.G.F; DINIZ, R.C. Plantas aromáticas e medicinais: cultivo e utilização. Londrina: IAPAR, 2008.

ROSA, G.; CÂMARA, S. G.; BÉRIA, J. U. Representações e intenção de uso da fitoterapia na atenção básica à saúde. Gien Saude Golet, v. 16, n. 1, p. 311-318, 2011.

RUIZ, A. L. T. G. et al. Farmacologia e toxicologia de Peumus boldus e Baccharis genistellóides. Rev Bras Farmacologn, v. 18, n. 2, p. 295-300, 2008.

SACRAMENTO, H. T. Legislação para produção, comercialização e uso de plantas medicinais. In: JORNADA PAULISTA DE PLANTAS MEDICINAIS, 5., 2001. Botucatu. Anais... Botucatu: UNESP, 2001. p. 33.

SAMPAIO, L. A. et al. Percepção dos enfermeiros da estratégia saúde da família sobre o uso da fitoterapia. Rev. Min. Enferm., Crato, v. 17, n. 1, p.77-84, jan./ mar. 2013.

SILVA, R. J. S. et al. Prevalência e fatores associados à percepção negativa da saúde em pessoas idosas no Brasil. Rev. Bras. Epi. v. 15, n. 1, p. 49-62, 2012

TEIXEIRA, A. $\mathrm{H}$ et al. Conhecimento popular sobre o uso de plantas medicinais no município de Sobral-Ceará, Brasil. SANARE, Sobral, v. 13, n. 1, p. 23-28, jan. 2014.

THIAGO, S. C. S.; TESSER, C. D. Percepção de médicos da Estratégia de Saúde da Família sobre terapias complementares. Rev. Saúde Pública, Florianópolis, v. 45, n. 2, p. 249-257, ago. 2010.

TITONELLI, N. A. A. et al. O uso de plantas medicinais como recurso terapêutico: das influências da formação profissional às implicações éticas e legais de sua aplicabilidade como extensão da prática de cuidar realizada pela enfermeira. Rev. Latino-Am. Enfermagem, v. 14, n. 3, p.316-323 2006 .

VEIGA, J et al, Plantas medicinais: cura segura? Quim. Nova, Rio de Janeiro, v. 28, n. 3, p. 519-528, fev. 2005.

WORLD HEALth ORGANIZATION. Tradicional Medicine Strategy 2002-2005. Genebra: WHO, 2002. 65 p. 